\title{
Enhanced Antibacterial Activity of Ag Nanoparticle-Decorated ZnO Nanorod Arrays
}

\author{
Jian Shang $\mathbb{D}^{1}{ }^{1}$ Ye Sun $\mathbb{D}^{2}{ }^{2}$ Teng Zhang $\mathbb{D}^{1},{ }^{1}$ Zhen Liu, ${ }^{1}$ and Hong Zhang ${ }^{2}$ \\ ${ }^{1}$ Department of Orthopedics, First Affiliated Hospital of Harbin Medical University, Harbin 150001, China \\ ${ }^{2}$ Condensed Matter Science and Technology Institute, School of Science, Harbin Institute of Technology, Harbin 150080, China
}

Correspondence should be addressed to Ye Sun; sunye@hit.edu.cn and Teng Zhang; 1142099451@qq.com

Received 15 October 2018; Revised 9 January 2019; Accepted 11 February 2019; Published 30 April 2019

Academic Editor: Angelo Taglietti

Copyright (c) 2019 Jian Shang et al. This is an open access article distributed under the Creative Commons Attribution License, which permits unrestricted use, distribution, and reproduction in any medium, provided the original work is properly cited.

\begin{abstract}
Silver $(\mathrm{Ag})$ has broad-spectrum antibacterial properties and is widely used in various fields, including in antibacterial coatings for orthopedic implants. For reasons of cost and cytotoxicity, improvement of the antibacterial efficiency of Ag is necessary. The scientific community has also shown a strong enthusiasm in this research area. In this paper, ZnO nanorod arrays were prepared on a titanium (Ti) substrate by seed-assisted hydrothermal method and Ag nanoparticles were deposited by magnetron sputtering to obtain $\mathrm{Ag}$ nanoparticle-decorated $\mathrm{ZnO}$ nanorod arrays ( $\mathrm{ZnO}$ nanorods/Ag nanoparticles). The antibacterial properties of $\mathrm{ZnO}$ nanorods/Ag nanoparticles against Pseudomonas aeruginosa were systematically studied by agar diffusion method and were compared with other samples such as $\mathrm{ZnO}$ nanorod arrays and $\mathrm{ZnO}$ seed layer/Ag nanoparticles. The experimental results showed that $\mathrm{ZnO}$ nanorods/Ag nanoparticles displayed significantly higher antibacterial properties against Pseudomonas aeruginosa than other samples, including $\mathrm{ZnO}$ nanorod arrays and $\mathrm{ZnO}$ seed layer/Ag nanoparticles. These superior antibacterial properties originated predominantly from the morphological structure of $\mathrm{ZnO}$ nanorods, which optimized the particle size and distribution of Ag nanoparticles, greatly improving their antimicrobial efficiency. The synergistic antibacterial properties of $\mathrm{Ag}$ nanoparticles and $\mathrm{ZnO}$ nanorods make $\mathrm{Ag}$ nanoparticle-decorated $\mathrm{ZnO}$ nanorod arrays a promising candidate for antibacterial coating of orthopedic implants.
\end{abstract}

\section{Introduction}

In orthopedics, implant-related postoperative infection is a serious complication with a high incidence rate of $1 \%-4 \%$ [1]. In recent years, the excessive use of antibiotics has led to a large number of drug-resistant strains, increasing the postoperative infection rate annually. To curb this and reduce antibiotics abuse, the research on antibacterial coating materials for orthopedic implants has received much attention in the scientific community.

Silver (Ag) nanoparticles have broad-spectrum antibacterial activities and good inhibitory effects on Gram-positive and Gram-negative bacteria, fungi, virus, and cancer cells [2-6], making them one of the most important antimicrobial materials. While increasing the amount of Ag nanoparticle used is the most straightforward method of enhancing the antibacterial properties of Ag-based antibacterial coatings, this method falls short in many aspects. First, the cost of Ag is relatively high. Second, $\mathrm{Ag}$ nanoparticles tend to agglomerate when they are present in large numbers, which reduces antibacterial efficacy [7]. The homogeneous distribution of Ag nanoparticles can produce small size and high surfaceto-volume ratio of Ag nanoparticles, which can improve the antibacterial effect. In particular, higher Ag dosage may have toxic or side effects on human cells and tissues. For example, overdose of $\mathrm{Ag}$ nanoparticles has been reported to suppress the human immune system [8] and induce the production of cytotoxins that damage fibrous tissues in the human lungs [9]. In this sense, improving the antibacterial efficiency of Ag nanoparticles has become a focus of antibacterial coating research. In contrast, zinc oxide $(\mathrm{ZnO})$ has good biocompatibility [10] and low cytotoxicity [11] and promotes the secretion of collagen by osteoblasts and the mineralization of the extracellular matrix [12]. In recent years, $\mathrm{ZnO}$ nanomaterials have been found to have good antibacterial activities [13-15] and have attracted much attention as antibacterial materials 
because of their low cost, simple preparation, high biocompatibility, and abundant morphological possibilities that are well controllable. A number of $\mathrm{Ag} / \mathrm{ZnO}$ materials with good antibacterial properties have been prepared by combining the two [16-21]. Recent reported studies focus on the preparation of different types of $\mathrm{Ag} / \mathrm{ZnO}$ samples, such as $\mathrm{Ag} / \mathrm{ZnO}$ nanocomposite [18], Ag-containing $\mathrm{ZnO}$ films [20], and Agdoped $\mathrm{ZnO}$ nanoparticles [21]. Most of the work is to enhance the antibacterial activity by changing the content or concentration of $\mathrm{Ag}[20,21]$. Although $\mathrm{Ag} / \mathrm{ZnO}$ has been reported to improve the antibacterial activity, little in-depth study has been conducted on how to optimize the antibacterial efficiency of $\mathrm{Ag}$ by using the synergistic antibacterial effect of $\mathrm{Ag}$ and $\mathrm{ZnO}$.

In this study, $\mathrm{ZnO}$ nanorod arrays were fabricated on $\mathrm{Ti}$ wafer by a modified seed-assisted hydrothermal method [22] and Ag nanoparticles were deposited by magnetron sputtering to develop $\mathrm{Ag}$ nanoparticle-decorated $\mathrm{ZnO}$ nanorod arrays [23]. The morphology and composition of the samples were characterized by SEM, XRD, and other techniques. The antibacterial properties of $\mathrm{ZnO}$ nanorods/Ag nanoparticles and other samples such as $\mathrm{ZnO}$ nanorod arrays and $\mathrm{ZnO}$ seed layer/Ag nanoparticles against Pseudomonas aeruginosa were studied by agar diffusion. The results of this study confirm the excellent antibacterial properties of $\mathrm{ZnO}$ nanorods/Ag nanoparticles and reveal the mechanism of their synergic antibacterial activities.

\section{Materials and Methods}

\subsection{Sample Preparation}

2.1.1. Preparation of the $\mathrm{ZnO}$ Seed Layer. The $\mathrm{ZnO}$ seed layer was fabricated on $10 \mathrm{~mm} \times 10 \mathrm{~mm}$ titanium (Ti) wafer by sputtering the $\mathrm{ZnO}$ target. The deposition of the seed layer was conducted under the following conditions: temperature of $400^{\circ} \mathrm{C}$, oxygen flow rate of $10 \mathrm{sccm}$, argon flow rate of $40 \mathrm{sccm}$, working pressure of $1 \mathrm{~Pa}$, and deposition time of $45 \mathrm{~s}$.

2.1.2. Preparation of $\mathrm{ZnO}$ Nanorods. Two types of $\mathrm{ZnO}$ nanorods were hydrothermally prepared on the $\mathrm{ZnO}$ seed layer with zinc nitrate $\left(\mathrm{Zn}\left(\mathrm{NO}_{3}\right)_{2}\right) \cdot\left(6 \mathrm{H}_{2} \mathrm{O}, 99 \%\right.$, Sinopharm $)$ and hexamethylenetetramine (HMT) $\left(\mathrm{C}_{6} \mathrm{H}_{12} \mathrm{~N}_{4}, 99 \%\right.$, Aladdin) as precursors. The first type of nanorods was synthesized by warming $50 \mathrm{~mL}$ each of $0.1 \mathrm{M} \mathrm{Zn}\left(\mathrm{NO}_{3}\right)_{2}$ and $\mathrm{HMT}$ at $90^{\circ} \mathrm{C}$ for $0.5 \mathrm{~h}$ and mixing the two solutions in a $130 \mathrm{~mL}$ glass bottle. The Ti/seed layer sample was immersed in this mixture, and the glass bottle was sealed. After reacting for $3 \mathrm{~h}$ at $90^{\circ} \mathrm{C}$, the sample was washed with deionized water and anhydrous ethanol. This $\mathrm{Ti} / \mathrm{ZnO}$ nanorod sample was named "SG," in which the amount of $\mathrm{ZnO}$ was approximately $500 \mu \mathrm{g} / \mathrm{cm}^{2}$. The second type of nanorod was prepared in the same manner, except for using $0.2 \mathrm{M} \mathrm{Zn}\left(\mathrm{NO}_{3}\right)_{2}$ and HMT solution.

2.1.3. Preparation of Ag Nanoparticle-Decorated $\mathrm{ZnO}$ Nanorod Arrays. Ag nanoparticles were deposited on the two types of $\mathrm{ZnO}$ nanorod arrays by radio frequency (RF) magnetron sputtering at room temperature. An RF power of $70 \mathrm{~W}$ was used for deposition. The deposition process took place at an argon flow rate of $40 \mathrm{sccm}$ and working pressure of $1 \mathrm{~Pa}$ for $30 \mathrm{~s}$. The amount of silver deposited was about $13 \mu \mathrm{g} / \mathrm{cm}^{2}$. The two types of $\mathrm{ZnO}$ nanorod/Ag nanoparticle samples obtained were named "SF" and "SE."

2.2. Sample Characterization. The structural properties of the resultant samples were investigated using an X-ray diffractometer (XRD) (PANalytical, X'Pert Pro) with $\mathrm{Cu} \mathrm{K} \alpha$ $(\lambda=0.154 \mathrm{~nm})$ radiation with a $2 \theta$ scanning range of $20^{\circ}$ $50^{\circ}$. The surface morphology was examined using scanning electron microscopy (SEM) (FEI Quanta 200F) with an accelerating voltage of $20 \mathrm{kV}$. An atomic force microscopy (AFM) (Bruker, MultiMode 8) was used for the analysis of the grain diameter.

2.3. Testing the Antibacterial Activity of the Samples. To study in depth the antibacterial properties of $\mathrm{ZnO}$ nanorods/Ag nanoparticles, tests were also performed on Ti wafer, hereafter referred to as $\mathrm{SA}, \mathrm{Ti} / \mathrm{Ag}$ nanoparticles $(\mathrm{SB}), \mathrm{Ti} / \mathrm{ZnO}$ seed layer (SC), $\mathrm{Ti} / \mathrm{ZnO}$ seed layer/Ag nanoparticles (SD), and $\mathrm{Ti} / \mathrm{ZnO}$ nanorods (SG). The $\mathrm{ZnO}$ seed layers and Ag nanoparticles were prepared in the same manner in all samples.

Our antibacterial activity test has been approved by the ISO15189 procedure. The antibacterial effectiveness of the samples was studied by agar diffusion [19, 24, 25]. Pseudomonas aeruginosa (ATCC2785, a Gram-negative bacterium) was used as the prototypical clinical bacteria with blood agar media (Columbia). Pseudomonas aeruginosa was inoculated on blood agar medium and incubated at $37^{\circ} \mathrm{C}$ for 24 hours. Then some colonies were diluted with $0.9 \%$ physiological saline to prepare $10^{8} \mathrm{cfu} \cdot \mathrm{mL}^{-1}$ suspension. This suspension was inoculated into two identical nutrient agar media N1 and N2. SA, SB, SC, and SD samples were placed into medium N1, and SE, SF, and SG sample were placed into medium N2. The culture media with the respective samples were incubated at $37^{\circ} \mathrm{C}$ for $48 \mathrm{~h}$ in an incubator. The size of the inhibition zone was measured by vernier caliper in millimeters $(\mathrm{mm})$.

\section{Results and Discussion}

The crystal structure of the samples was first characterized by $\mathrm{XRD}$ to prove the successful preparation of $\mathrm{ZnO}$ nanorods/Ag nanoparticles. Highly similar XRD spectra were obtained on the $\mathrm{ZnO}$ nanorods/Ag nanoparticles samples. As shown in the XRD spectrum of the SF sample in Figure 1, the characteristic peaks of $\mathrm{ZnO}$ (100), (002), (101), and (102) are correspond to the wurtzite hexagonal phase of $\mathrm{ZnO}$ (JCPDS 03-0888). The diffraction peaks appeared at $2 \theta=38.1^{\circ}$ corresponding to the cubic-phase $\mathrm{Ag}$ (111) (JCPDS 03-0921). Notably, a sharp, strong, and dominant peak of $\mathrm{ZnO}(002)$ centered at $34.4^{\circ}$ was observed, which was significantly higher than the other $\mathrm{ZnO}$ diffraction peaks, revealing the preferential $<002>$ orientation of this sample [26].

In addition, additional flat and broad peaks appeared at $2 \theta=35.5^{\circ}$ and $40.5^{\circ}$, which have originated from the 


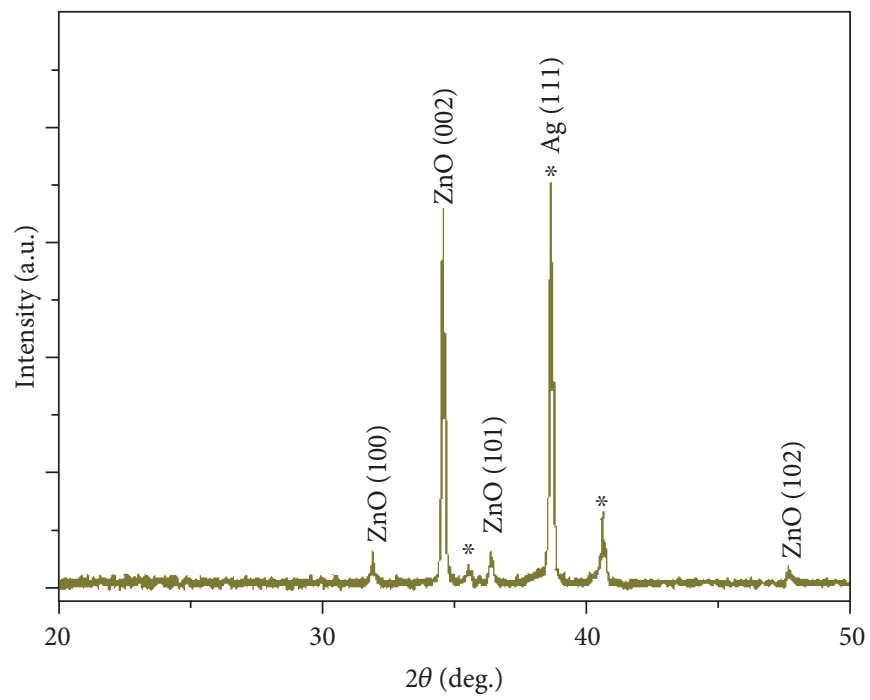

Figure 1: X-ray diffraction spectrum of the $\mathrm{ZnO}$ nanorod/Ag nanoparticle (SF) sample. The peaks marked with an asterisk are associated with the titanium alloy plate.

titanium alloy plate substrate. These results indicated the formation of highly crystalline $\mathrm{ZnO}$ and $\mathrm{Ag}$ in this work.

The SEM images of $\mathrm{ZnO}$ nanorod/Ag nanoparticle samples are displayed in Figure 2. Figure 2(a) is the top-view SEM image of SF at low magnification, in which numerous uniformly distributed hexagonal prism-like nanorods can be seen. This is consistent with the typical morphology of $\mathrm{ZnO}$ nanorods reported in the literature [27-29]. Most nanorods grow perpendicular to the Ti substrate, forming highly $<002>$ oriented nanorod arrays, as also shown in the XRD data. These nanorods were mostly even in diameter, with an average diameter of about $85 \mathrm{~nm}$. Signs of Ag nanoparticles are not evident in the low-magnification SEM image of Figure 2(a), as the number and size of Ag nanoparticles are much smaller than those of $\mathrm{ZnO}$ nanorods. The surface of pure $\mathrm{ZnO}$ nanorods was smooth. In the high-magnification top-view SEM image of SF (Figure 2(b)), we can clearly observe the rough surface of nanorods, which corresponds to Ag nanoparticles (AgNPs) with uniform sizes, confirming the successful fabrication of Ag nanoparticle-modified $\mathrm{ZnO}$ nanorod arrays. The morphology of SE samples is also characterized by low- and high-magnification SEM images (Figures 2(c) and 2(d), respectively). In Figure 2(c), SE also contains a large number of hexagonal prism nanorods with preferential orientation. They had a diameter of approximately $135 \mathrm{~nm}$, which is significantly greater than the diameter of the nanorods in SF samples, as the $\mathrm{ZnO}$ nanorods in SE were prepared from precursors with higher concentrations.

Figure 2(d) shows that the surface of nanorods in SE samples is also covered by nanoparticles. As indicated in these SEM results, the sizes of $\mathrm{Ag}$ nanoparticles in both $\mathrm{ZnO}$ nanorod/Ag nanoparticle samples were very small, in the range of 10-20 nm. The nanoparticles formed here were significantly smaller than those in the $\mathrm{Ti} / \mathrm{ZnO}$ seed layer/Ag nanoparticle samples, which were measured by AFM to have sizes of 40-100 nm (Figure 3 ). These results confirmed the successful preparation of highly crystalline $\mathrm{ZnO}$ nanorods/Ag nanoparticles and that $\mathrm{ZnO}$ nanorod arrays had a substantial effect on the size and distribution of $\mathrm{Ag}$ nanoparticles.

The antimicrobial activity of the samples was determined by the size of the Pseudomonas aeruginosa inhibition zone formed. Figure 4 shows the bacteria inhibition rings produced by SA, SB, SC, SD, SE, SF, and SG samples after $24 \mathrm{~h}$ of culturing in an incubator at $37^{\circ} \mathrm{C}$. In medium N1, no distinct bacterial inhibition zone is observed around samples SA (Ti wafer), SB (Ti/Ag nanoparticles), and SC (Ti/ZnO seed layer), while a small bacterial inhibition zone is seen around sample SD ( $\mathrm{Ti} / \mathrm{ZnO}$ seed layer/Ag nanoparticles). These results showed that the $\mathrm{ZnO}$ seed layer or Ag nanoparticles on the Ti substrate alone had no appreciable antibacterial activity against Pseudomonas aeruginosa but a combination of the two could give synergic antibacterial effect. Sample SB should have antibacterial activity, but we did not see the obvious inhibition zone in our work. It may be due to the agglomeration of Ag nanoparticles, which weakens the antibacterial effect and results in the low concentration of $\mathrm{Ag}$ ions released into the surrounding environment.

In the N2 medium, noticeable bacterial inhibition zones were formed around the SE, SF, and SG samples. The inhibition zone formed by SG ( $\mathrm{ZnO}$ nanorods) was smaller and had a diameter of $11.5 \mathrm{~mm}$, while those by SE and SF (two $\mathrm{ZnO}$ nanorod/Ag nanoparticle samples) were larger and had diameters of $13.1 \mathrm{~mm}$ and $13.2 \mathrm{~mm}$, respectively. The antibacterial properties of $\mathrm{ZnO}$ and $\mathrm{Ag}$ were then studied in depth by comparing the antibacterial actions of different samples. First, the antibacterial properties of SG ( $\mathrm{ZnO}$ nanorods) were similar to those of SD ( $\mathrm{ZnO}$ seed layer/Ag nanoparticles). The amount of $\mathrm{ZnO}$ in the SG sample was about $500 \mu \mathrm{g} / \mathrm{cm}^{2}$, while the amount of Ag deposited in the SD sample was about $13 \mu \mathrm{g} / \mathrm{cm}^{2}$. Therefore, $\mathrm{ZnO}$ nanorods, although had some antibacterial effects on Pseudomonas 


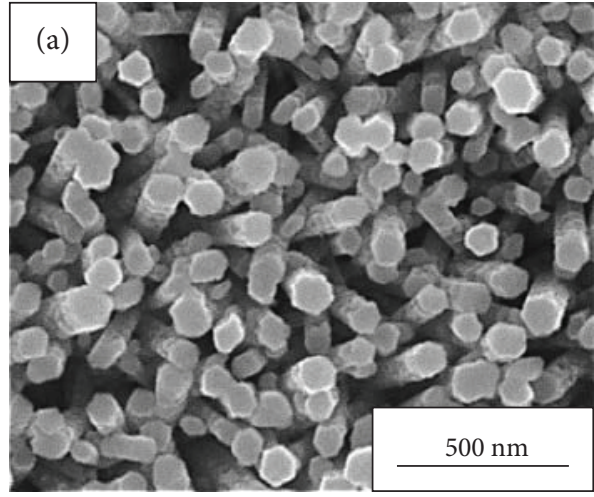

(a)

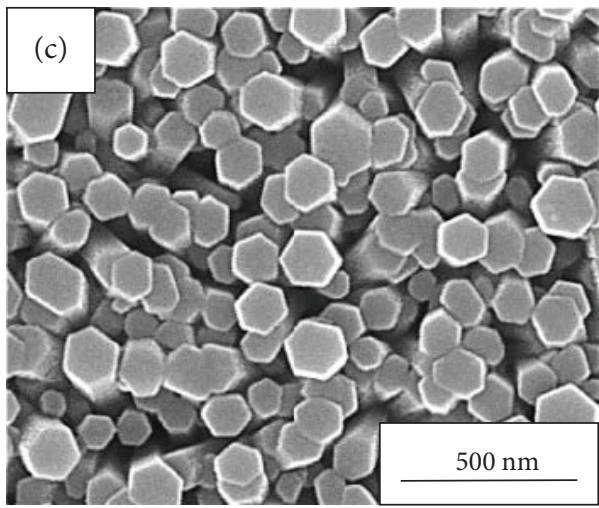

(c)

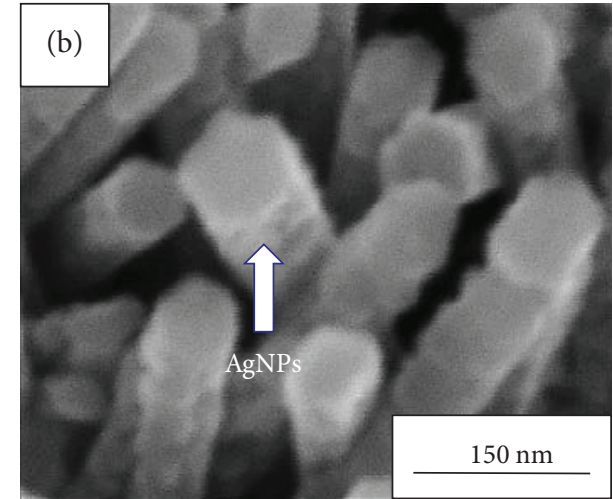

(b)

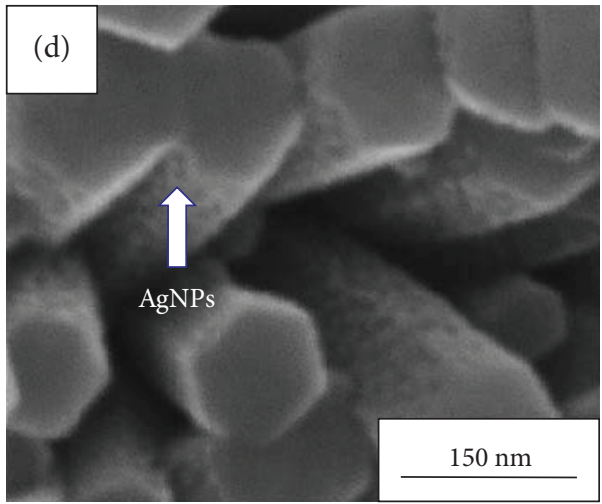

(d)

FIgURE 2: Scanning electron microscopy images of the SF (a, b) and SE (c, d) samples.

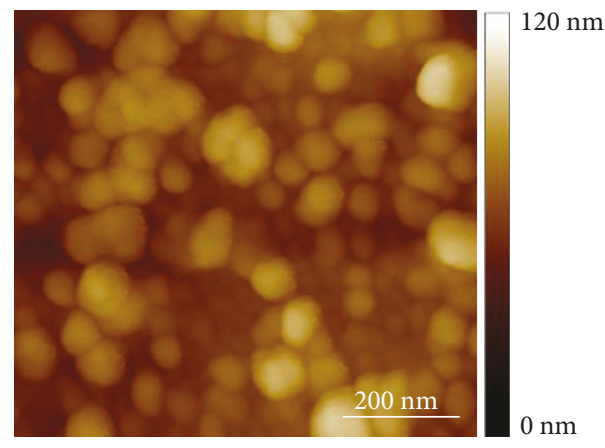

Figure 3: Atomic force microscopy image of the SD sample.

aeruginosa, were much less capable of doing so than $\mathrm{Ag}$ nanoparticles [30]. When the antibacterial functions of SF ( $\mathrm{ZnO}$ nanorods/Ag nanoparticles) and $\mathrm{SD}$ ( $\mathrm{ZnO}$ seed layer/Ag nanoparticles) were compared, the far more superior antibacterial activities of the former were clearly not only caused by the difference between $\mathrm{ZnO}$ nanorods and the $\mathrm{ZnO}$ seed layer in this respect. Hence, the Ag nanoparticles on $\mathrm{ZnO}$ nanorods had higher antibacterial capability than those on the $\mathrm{ZnO}$ seed layer, although the same total amount of $\mathrm{Ag}$ was present in both cases. As shown in Figure 2(b), the particle size of Ag nanoparticles in the SF sample ranges from 10 to $20 \mathrm{~nm}$, much less than the $40-100 \mathrm{~nm}$ size of Ag nanoparticles on the seed layer (Figure 3). This could be the reason for the higher antibacterial activities of Ag nanoparticles on
$\mathrm{ZnO}$ nanorods, i.e., the morphology and large surface area of the nanorods make it possible for Ag nanoparticles to distribute over a greater area on the nanorods and have a larger surface area, facilitating the antimicrobial action of the latter [31]. In addition, the key mechanism for Ag nanoparticles to exert antibacterial activity is through the release of $\mathrm{Ag}$ ions. As the size of nanoparticles decreases, the surface area will increase. The release rate of $\mathrm{Ag}$ ions is proportional to the surface area of the particles, so the release rate of $\mathrm{Ag}$ ions from smaller nanoparticles is faster than that of larger nanoparticles, which improves the antibacterial properties of $\mathrm{Ag}$ nanoparticles [32, 33]. On the contrary, although SE samples definitely contain more $\mathrm{ZnO}$ than $\mathrm{SF}$ samples, the antibacterial activities of the two come very close, with SE being even lower by a small amount. This is another proof of Ag nanoparticles as the primary origin of antibacterial functions in $\mathrm{ZnO}$ nanorods/Ag nanoparticles, despite some antibacterial properties being detected in $\mathrm{ZnO}$ nanorods. In contrast, $\mathrm{SB}$ (Ti/Ag nanoparticles) samples showed almost no antibacterial effect. The above evidence leads to the conclusion that the synergic antibacterial activities of $\mathrm{ZnO}$ nanorods and $\mathrm{Ag}$ nanoparticles were mainly derived from the morphology and structure of $\mathrm{ZnO}$ nanorods, which optimized the size and distribution of Ag nanoparticles and greatly improved their antibacterial efficiency.

Figure 5 shows the changes in the inhibition zones of SD, SE, SF, and SG cultured for different durations in an incubator at $37^{\circ} \mathrm{C}$. Strong antibacterial activities are seen in all four 


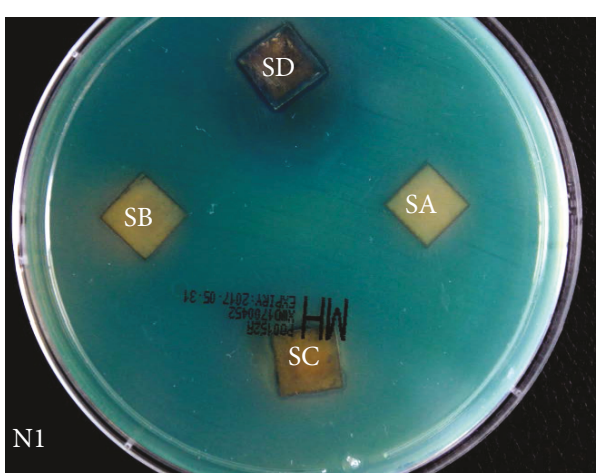

(a)

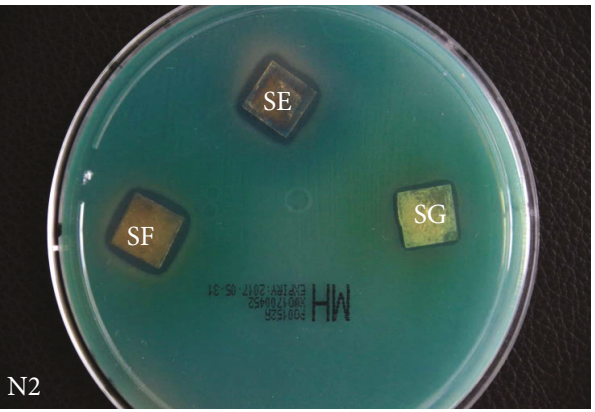

(b)

FIgUre 4: The size of the inhibition zone of Pseudomonas aeruginosa after $24 \mathrm{~h}$ of culture.

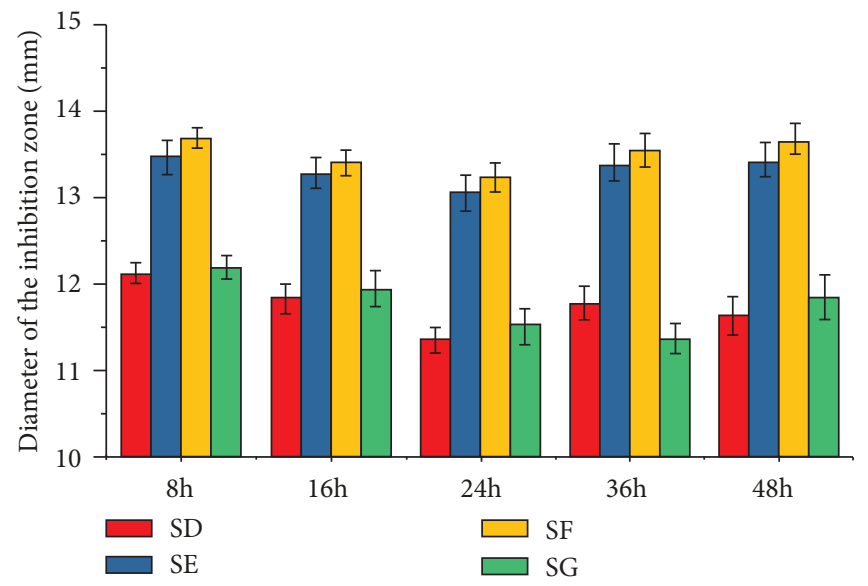

FIGURE 5: The inhibition zone test of the SD, SE, SF, and SG samples at different times.

samples within $48 \mathrm{~h}$. The diameters of the inhibition zones at $48 \mathrm{~h}$ were similar to those at $8 \mathrm{~h}$, which may be related to the diffusion of $\mathrm{Ag}$ ions in the agar media, and the antibacterial activity mainly depended on the active surface area of $\mathrm{Ag}$ in the agar media [34]. The bacterial inhibition zones of SD and SG had similar diameters, as well as those of SF and SE samples. The diameters of the inhibition zones of SE samples at $8,16,24,36$, and 48 hours were $13.5,13.3,13.1,13.4$, and $13.5 \mathrm{~mm}$, respectively. Similarly, the inhibition zones of SF samples were $13.7,13.4,13.2,13.5$, and $13.7 \mathrm{~mm}$, respectively. With prolonged incubation, the diameters of the inhibition zones formed by SF and SE were maintained at 13.1$13.7 \mathrm{~mm}$, proving the superior long-term antibacterial properties of $\mathrm{ZnO}$ nanorods/Ag nanoparticles.

\section{Conclusions}

In summary, $\mathrm{ZnO}$ nanorods/Ag nanoparticles were prepared on a Ti substrate using both gas- and liquid-phase methods. Their excellent synergic antibacterial properties were confirmed with Pseudomonas aeruginosa as the target. Comparison with other samples was also carried out as verification. Although $\mathrm{ZnO}$ nanorods alone also have some antibacterial effects, the superiority of $\mathrm{ZnO}$ nanorods/Ag nanoparticles in this respect was mainly derived from the morphological structure of $\mathrm{ZnO}$ nanorods, which optimized the size and distribution of Ag nanoparticles and greatly enhanced their antibacterial efficacy. Improving the antibacterial efficiency of Ag nanoparticles reduces the cytotoxicity induced by high dosage of $\mathrm{Ag}$ and lowers the cost of antibacterial coating. Because of its synergic antibacterial activity, this type of $\mathrm{ZnO}$ nanorod/Ag nanoparticle material will have broad usage prospects as antibacterial coatings on orthopedic implants. In addition, this work provides a novel route for the synthesis of Ag-based coatings with higher antibacterial efficiency.

\section{Data Availability}

The data used to support the findings of this study are included within the article.

\section{Conflicts of Interest}

The authors declare that they have no conflicts of interest.

\section{Acknowledgments}

This work was financially supported by the Science and Technology Research Project of Heilongjiang Provincial Education Department (12541344). 


\section{References}

[1] B. Laure, J. M. Besnier, A. M. Bergemer-Fouquet et al., "Effect of hydroxyapatite coating and polymethyimemacrylate on stainless steel implant site infection with Staphylococcus epidermids in a sleep model," Journal of Biomedical Materials Research Part A, vol. 84A, no. 1, pp. 92-98, 2008.

[2] I. Sondi and B. Salopek-Sondi, "Silver nanoparticles as antimicrobial agent: a case study on E.coli as a model for Gramnegative bacteria," Journal of Colloid and Interface Science, vol. 275, no. 1, pp. 177-182, 2004.

[3] W.-R. Li, X. B. Xie, Q. S. Shi, H. Y. Zeng, Y. S. OU-Yang, and Y. B. Chen, "Antibacterial activity and mechanism of silver nanoparticles on Escherichia coli," Applied Microbiology and Biotechnology, vol. 85, no. 4, pp. 1115-1122, 2010.

[4] Z. K. Xia, Q. H. Ma, S. Y. Li et al., "The antifungal effect of silver nanoparticles on Trichosporon asahii," Journal of Microbiology, Immunology and Infection, vol. 49, no. 2, pp. 182-188, 2016.

[5] S. Gaikwad, A. Ingle, A. Gade et al., "Antiviral activity of mycosynthesized silver nanoparticles against herpes simplex virus and human parainfluenza virus type 3," International Journal of Nanomedicine, vol. 8, no. 1, pp. 4303-4314, 2013.

[6] M. Jeyaraj, M. Rajesh, R. Arun et al., "An investigation on the cytotoxicity and caspase-mediated apoptotic effect of biologically synthesized silver nanoparticles using Podophyllum hexandrum on human cervical carcinoma cells," Colloids and Surfaces B: Biointerfaces, vol. 102, no. 1, pp. 708-717, 2013.

[7] F. Ghilini, M. C. Rodríguez González, A. G. Miñán et al., "Highly stabilized nanoparticles on poly-L-lysine-coated oxidized metals: a versatile platform with enhanced antimicrobial activity," ACS Applied Materials \& Interfaces, vol. 10, no. 28, pp. 23657-23666, 2018.

[8] W. H. de Jong, L. T. M. van der Ven, A. Sleijffers et al., "Systemic and immunotoxicity of silver nanoparticles in an intravenous 28 days repeated dose toxicity study in rats," Biomaterials, vol. 34, no. 33, pp. 8333-8343, 2013.

[9] P. V. AshaRani, G. Low Kah Mun, M. P. Hande, and S. Valiyaveettil, "Cytotoxicity and genotoxicity of silver nanoparticles in human cells," ACS Nano, vol. 3, no. 2, pp. 279-290, 2009.

[10] K. Huo, X. Zhang, H. Wang, L. Zhao, X. Liu, and P. K. Chu, "Osteogenic activity and anti-bacterial effects on titanium surfaces modified with $\mathrm{Zn}$-incorporated nanotube arrays," Biomaterials, vol. 34, no. 13, pp. 3467-3478, 2013.

[11] G. J. Nohynek, E. Antignac, T. Re, and H. Toutain, "Safety assessment of personal care products/cosmetics and their ingredients," Toxicology and Applied Pharmacology, vol. 243, no. 2, pp. 239-259, 2010.

[12] G. Jin, H. Cao, Y. Qiao, F. Meng, H. Zhu, and X. Liu, “Osteogenic activity and antibacterial effect of zinc ion implanted titanium," Colloids and Surfaces B: Biointerfaces, vol. 117, no. 9, pp. 158-165, 2014.

[13] G. Applerot, N. Perkas, G. Amirian, O. Girshevitz, and A. Gedanken, "Coating of glass with $\mathrm{ZnO}$ via ultrasonic irradiation and a study of its antibacterial properties," Applied Surface Science, vol. 256, no. 3, pp. S3-S8, 2009.

[14] K. H. Tam, A. B. Djurišić, C. M. N. Chan et al., “Antibacterial activity of $\mathrm{ZnO}$ nanorods prepared by a hydrothermal method," Thin Solid Films, vol. 516, no. 18, pp. 6167-6174, 2008.
[15] M. Ramani, S. Ponnusamy, and C. Muthamizhchelvan, "Preliminary investigations on the antibacterial activity of zinc oxide nanostructures," Journal of Nanoparticle Research, vol. 15, no. 4, p. 1557, 2013.

[16] S. Ghosh, V. S. Goudar, K. G. Padmalekha, S. V. Bhat, S. S. Indi, and H. N. Vasan, " $\mathrm{ZnO} / \mathrm{Ag}$ nanohybrid: synthesis, characterization, synergistic antibacterial activity and its mechanism," RSC Advances, vol. 2, no. 3, pp. 930-940, 2012.

[17] D. V. Ponnuvelu, S. P. Suriyaraj, T. Vijayaraghavan, R. Selvakumar, and B. Pullithadathail, "Enhanced cell-wall damage mediated, antibacterial activity of core-shell ZnO@Ag heterojunction nanorods against Staphylococcus aureus and Pseudomonas aeruginosa," Journal of Materials Science: Materials in Medicine, vol. 26, no. 7, p. 204, 2015.

[18] S. Wang, J. Wu, H. Yang, X. Liu, Q. Huang, and Z. Lu, “Antibacterial activity and mechanism of $\mathrm{Ag} / \mathrm{ZnO}$ nanocomposite against anaerobic oral pathogen Streptococcus mutans," Journal of Materials Science: Materials in Medicine, vol. 28, no. 1, p. 23, 2017.

[19] A. H. Shah, E. Manikandan, M. Basheer Ahmed, and V. Ganesan, "Enhanced bioactivity of $\mathrm{Ag} / \mathrm{ZnO}$ nanorods-a comparative antibacterial study (Sbds)," Journal of Nanomedicine \& Nanotechnology, vol. 4, no. 3, p. 1, 2013.

[20] T. Fu, F. Zhang, Z. Alajmi, S. Y. Yang, F. Wu, and S. L. Han, "Sol-gel derived antibacterial Ag-containing $\mathrm{ZnO}$ films on biomedical titanium," Journal of Nanoscience and Nanotechnology, vol. 18, no. 2, pp. 823-828, 2018.

[21] G. Y. Nigussie, G. M. Tesfamariam, B. M. Tegegne et al., “Antibacterial activity of Ag-doped $\mathrm{TiO}_{2}$ and Ag-doped $\mathrm{ZnO}$ nanoparticles," International Journal of Photoenergy, vol. 2018, Article ID 5927485, 7 pages, 2018.

[22] Y. Yin, Y. Sun, M. Yu et al., "Controlling the hydrothermal growth and the properties of $\mathrm{ZnO}$ nanorod arrays by pretreating the seed layer," RSC Advances, vol. 4, no. 84, pp. 44452-44456, 2014.

[23] A. S. M. Iftekhar Uddin and G. S. Chung, "A novel flexible $\mathrm{C}_{2} \mathrm{H}_{2}$ gas sensor based on Ag-ZnO nanorods on PI/PTFE substrate," in Proceedings Volume 9749, Oxide-based Materials and Devices VII; 97491T, San Francisco, CA, USA, February 2016.

[24] L. Lei, X. Liu, Y. Yin, Y. Sun, M. Yu, and J. Shang, "Antibacterial $\mathrm{Ag}-\mathrm{SiO}_{2}$ composite films synthesized by pulsed laser deposition," Materials Letters, vol. 130, pp. 79-82, 2014.

[25] S. C. Motshekga, S. S. Ray, M. S. Onyango, and M. N. B. Momba, "Microwave-assisted synthesis, characterization and antibacterial activity of $\mathrm{Ag} / \mathrm{ZnO}$ nanoparticles supported bentonite clay," Journal of Hazardous Materials, vol. 262, no. 262, pp. 439-446, 2013.

[26] Y. Yin, Y. Sun, M. Yu et al., "Arrays of nanorods composed of $\mathrm{ZnO}$ nanodots exhibiting enhanced UV emission and stability," Nanoscale, vol. 6, no. 18, pp. 10746-10751, 2014.

[27] Y. Yin, Y. Sun, M. Yu et al., "Reagent concentration dependent variations in the stability and photoluminescence of silicacoated ZnO nanorods," Inorganic Chemistry Frontiers, vol. 2, no. 1, pp. 28-34, 2015.

[28] Y. Yin, Y. Sun, M. Yu et al., "ZnO nanorod array grown on Ag layer: a highly efficient fluorescence enhancement platform," Scientific Reports, vol. 5, no. 1, article 8152, 2015.

[29] Y. Sun, N. A. Fox, D. J. Riley, and M. N. R. Ashfold, "Hydrothermal growth of $\mathrm{ZnO}$ nanorods aligned parallel to the 
substrate surface," Journal of Physical Chemistry C, vol. 112, no. 25, pp. 9234-9239, 2008.

[30] J. F. Hernández-Sierra, F. Ruiz, D. C. Cruz Pena et al., “The antimicrobial sensitivity of Streptococcus mutans to nanoparticles of silver, zinc oxide, and gold," Nanomedicine: Nanotechnology, Biology, and Medicine, vol. 4, no. 3, pp. 237-240, 2008.

[31] Y. Chen, W. H. Tse, L. Chen, and J. Zhang, "Ag nanoparticlesdecorated $\mathrm{ZnO}$ nanorod array on a mechanical flexible substrate with enhanced optical and antimicrobial properties," Nanoscale Research Letters, vol. 10, no. 1, p. 106, 2015.

[32] C. Marambio-Jones and E. M. V. Hoek, "A review of the antibacterial effects of silver nanomaterials and potential implications for human health and the environment," Journal of Nanoparticle Research, vol. 12, no. 5, pp. 1531-1551, 2010.

[33] M. Rai, A. Yadav, and A. Gade, "Silver nanoparticles as a new generation of antimicrobials," Biotechnology Advances, vol. 27, no. 1, pp. 76-83, 2009.

[34] M. Boswald, M. Girisch, J. Greil et al., “Antimicrobial activity and biocompatibility of polyurethane and silicone catheters containing low concentrations of silver: a new perspective in prevention of polymer-associated foreign-body-infections," Zentralblatt für Bakteriologie, vol. 283, no. 2, pp. 187-200, 1995. 


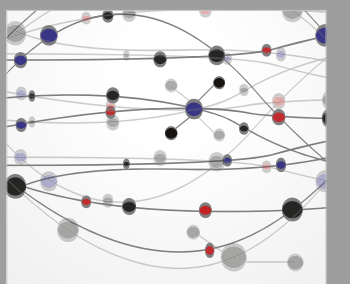

The Scientific World Journal
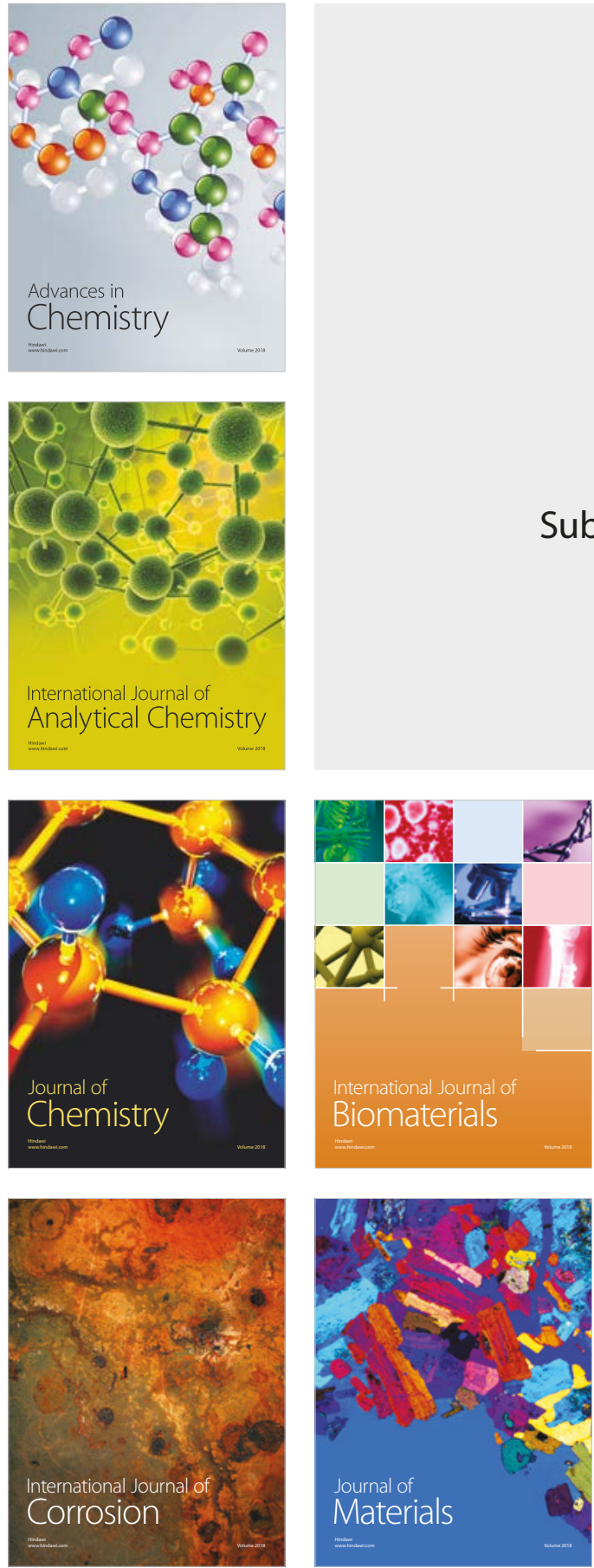

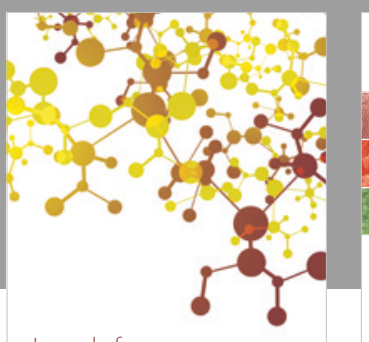

Journal of

Applied Chemistry
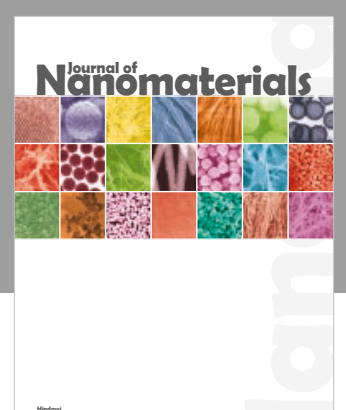

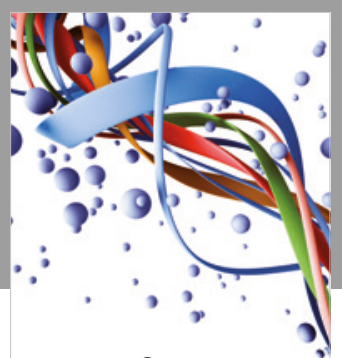

Scientifica

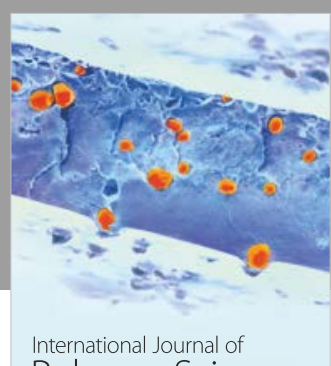

Polymer Science

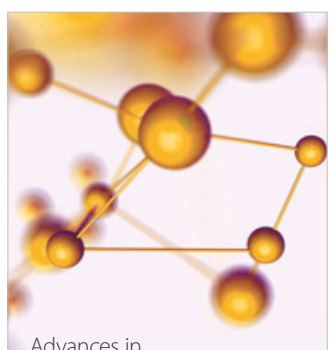

Physical Chemistry
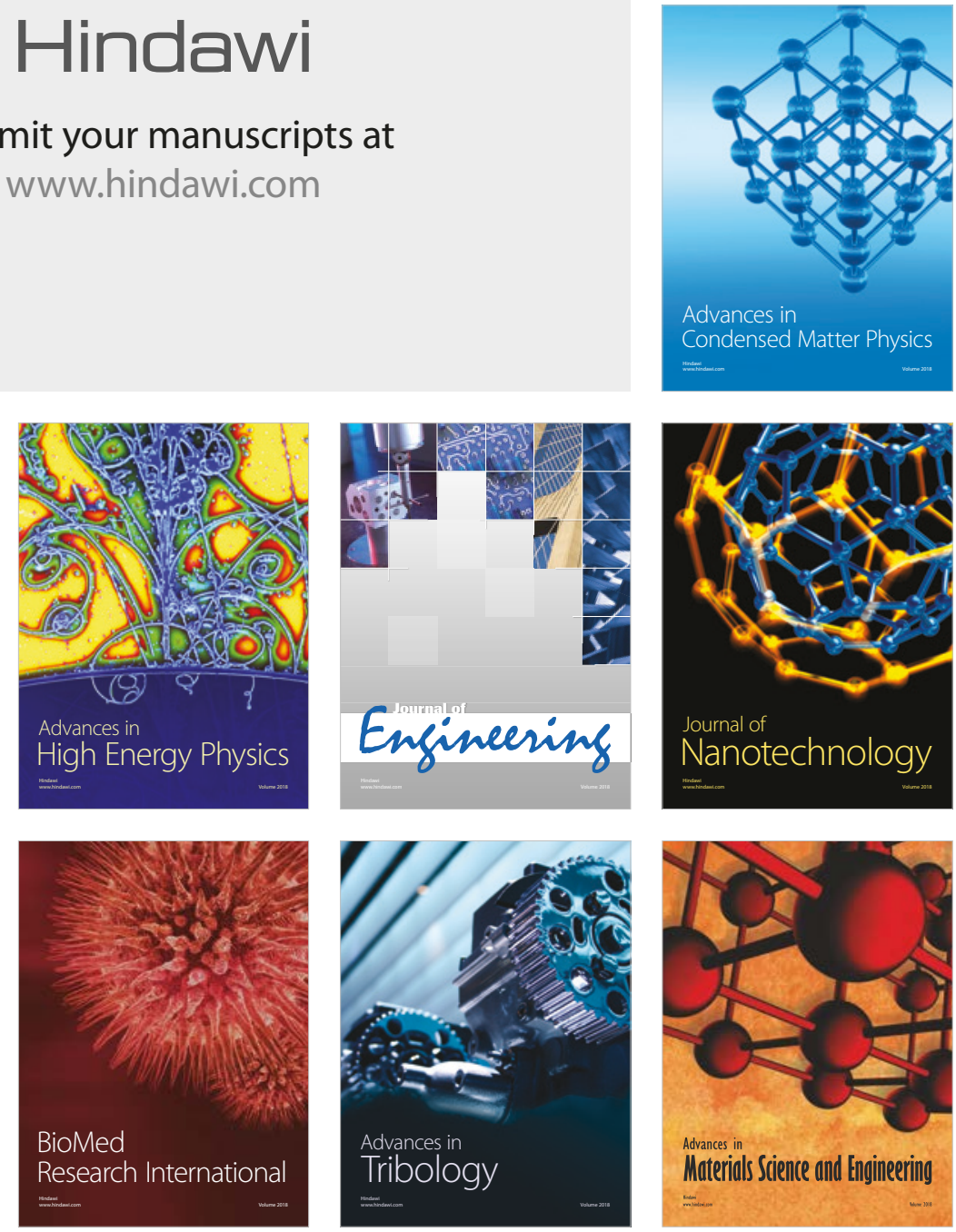